\title{
3D Magnetostatic Field Computation Based on Hybrid Mesh Integral Equation Method
}

\author{
Wubing Zhu ${ }^{1, \text { a }}$, Wenchun Zhao ${ }^{1, b}$, Jinwu Zhuang ${ }^{1, \mathrm{c}}$ and Shengdao Liu ${ }^{1, \mathrm{~d}}$ \\ ${ }^{1}$ Electrical Engineering, Naval University of Engineering, Wuhan China \\ a zhuwubing2014@hotmail.com, ${ }^{b}$ zhaowenchun1973@hotmail.com, \\ cdelubinavy1@263.net, d18986151073@189.cn
}

\begin{abstract}
Keywords: integral equation method, ferromagnetic object, hybrid mesh, 3D magnetostatic field Abstract. Accurate magnetostatic field computation for complex 3D ferromagnetic objects can be widely used to solve various engineering problems, while mesh quality is a key factor that can greatly affect the calculation precision. For complex shaped 3D objects, their mesh quality can be improved greatly by using hybrid mesh strategy with the advantages of hexahedral elements and tetrahedral elements. In this paper, a hybrid mesh integral equation method (IEM) for 3D magnetostatic field computation is realized based on mixed mesh with hexahedrons and tetrahedrons. Furthermore, computational accuracy of the proposed method is demonstrated by simulation and experimental examples with $2.10 \%$ and $3.20 \%$, respectively. Thus, foundations for the mixed mesh strategy for the complex shaped 3D objects and fast computational methods with hybrid mesh IEM are established.
\end{abstract}

\section{Introduction}

Induced magnetism which caused by external environmental magnetic field of carriers (such as ships, planes, etc) composed of ferromagnetic materials is one of main threats to the navigation security of naval vessels [1,2] and a key factor to restrict the development of high-precision magnetism measurement technology executed by magnetic sensor carriers [3]. In the field of ship degaussing [4,5], mastering the characteristics of vessels' induced magnetic field is an extremely important factor to realize the high-precision compensation of induced magnetic field. In terms of marine magnetic survey and aeromagnetic survey, induced magnetic field is one of the main interference sources [6,7]. Due to the complexity of geometric features of vehicles, the numerical methods such as Finite Element Method (FEM) [8], Boundary Element Method (BEM) [9], Integral Equation Method (IEM) [10,11], have been effective tools in calculating induced magnetism accurately. Compared with FEM and the BEM, IEM is more suitable for the open boundary magnetostatic field calculation avoiding the meshing of air region (or equivalent) [10-13].

In terms of induced magnetism numerical modelling of vehicles, a series of research developments have been achieved by using IEM.In Ref.[14], compared with the volume integral method, the computation of tetrahedron coupling coefficient matrix could be simpler and the integral singularity could be eliminated by using the surface integral calculation method, while the nonsymmetric and dense coefficient matrix of IEM models could result in low computation efficiency. Recently, accelerating approaches such as Adaptive Cross Approximation(ACA) [15], Fast Multipole Method [11,16] and Parallel Algorithm have been applied to overcome the disadvantage above and solve the large-scale magnetism computation problem successfully. Usually, tetrahedral elements and hexahedral elements can be used to discrete ferromagnetic objects and the two kind of mesh elements above have their advantages, respectively [19]. By meshing 3D objects with both tetrahedrons and hexahedrons, advantages of the two kinds of elements above could be combined. Induced magnetism computation based on hybrid mesh is realized with good accuracy in this article, which lays foundations for the mixed mesh strategy for the complex shaped 3D objects and fast computational methods for the proposed hybrid mesh IEM.

In Section 2, vector integral equation method based on mesh elements will be clearly introduced. In Section 3, theory for calculating the coupling coefficient matrixs of tetrahedral elements and 
hexahedral elements will be demonstrated in detail. Then in Section 4, magnetostatic field modeling of an irregular ferromagnetic object and a thin steel plate immerged into external magnetic field will be displayed. Finally, the magnetism field results of the two objects calculated by the hybrid mesh IEM will be compared with simulations with FEM software Flux3D and experimental measurements, respectively.

\section{Vector integral equation method}

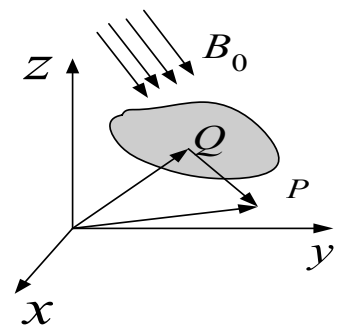

Fig. 1 Sketch of a magnetostatic computation

As shown in Fig. 1, according to IEM, when a ferromagnetic object is placed in a geomagnetic field, the induced magnetic flux density $\boldsymbol{B}$ at an arbitrary point $P$ outside the object can be expressed as [18]

$$
\boldsymbol{B}\left(\boldsymbol{r}_{P}\right)=-\frac{\mu_{0}}{4 \pi} \nabla_{P} \int_{V} \boldsymbol{M}\left(\boldsymbol{r}_{Q}\right) \cdot \nabla_{Q}\left(\frac{1}{\left|\boldsymbol{r}_{P Q}\right|}\right) d v_{Q}
$$

where $\boldsymbol{M}$ denotes the magnetization of the ferromagnetic object, $r_{P Q}$ represents the vector from the source point $Q$ to the field point $P, \mu_{0}$ represents the permeability of free space, $\nabla_{p}$ and $\nabla_{Q}$ represent the gradient operator to the field coordinate and the source coordinate, respectively, and $V$ represents the volume of the object.

To calculate the induced magnetic flux density $\boldsymbol{B}$ created by the ferromagnetic object, the object should be discretized into $N$ elements that may be tetrahedral in shape. Eq. (1) becomes,

$$
\boldsymbol{B}\left(\boldsymbol{r}_{\boldsymbol{P}}\right)=-\frac{\mu_{0}}{4 \pi} \sum_{i=1}^{N} \nabla_{\boldsymbol{P}} \int_{V_{i}} \boldsymbol{M}\left(\boldsymbol{r}_{Q}\right) \cdot \nabla_{Q}\left(\frac{1}{\left|\boldsymbol{r}_{\boldsymbol{P} Q}\right|}\right) \boldsymbol{d} \boldsymbol{v}_{Q}
$$

As far as the magnetization of each element is concerned, the constant, linear, and higher order shape functions can be used. For simplicity, the constant shape function is used in this computation, Then the field created by each volume element can be calculated using the equivalent surface distribution method [13],

$$
\boldsymbol{B}\left(\boldsymbol{r}_{\boldsymbol{P}}\right)=-\frac{\mu_{0}}{4 \pi} \sum_{i=1}^{N}\left(\nabla_{P} \int_{S_{i}} \frac{\boldsymbol{M}_{\boldsymbol{i}}\left(\boldsymbol{r}_{Q}\right) \boldsymbol{g}_{i}}{\left|\boldsymbol{r}_{P Q}\right|} d S_{Q}\right)
$$

where $S_{i}$ represents the surface area of the volume element and $\boldsymbol{n}_{\boldsymbol{i}}$ represents the direction normal to the surface. When computing the induced field created by an external magnetic field, the object is usually divided into a sufficient number of uniform elements. Considering the relationship $\boldsymbol{M}=\boldsymbol{B}\left(\mu_{r}-1\right) / \mu_{r}$ and each of the $N$ field points that are placed at the barycenter of the corresponding tetrahedral element, the following system can be obtained,

$$
\frac{\mu_{r j}}{4 \pi} \sum_{i=1}^{N} \frac{\mu_{r i}-1}{\mu_{r i}} \int_{s_{i}}\left(\boldsymbol{B}\left(\boldsymbol{r}_{Q_{i}}\right) \cdot \boldsymbol{n}_{i}\right) \frac{\boldsymbol{r}_{P Q_{i}}}{\left|\boldsymbol{r}_{P Q_{i}}\right|^{3}} d s_{Q}-\boldsymbol{B}\left(\boldsymbol{r}_{P_{j}}\right)=-\mu_{r j} \boldsymbol{B}_{0}\left(\boldsymbol{r}_{P_{j}}\right)
$$

where $i$ and $j$ represent the element numbers while $\mu_{r i}$ and $\mu_{r j}$ denote the relative permeability of respective tetrahedral elements. Compared with other strong magnetic fields, the geomagnetic field is so weak that the relative permeability $\mu_{r}$ can be treated as a constant. Eq. (4) can then be transformed into a matrix form as follows 


$$
\frac{\mu_{r j}}{4 \pi} \sum_{i=1}^{N} \frac{\mu_{r i}-1}{\mu_{r i}} \boldsymbol{S}_{P_{j} Q_{i}} \boldsymbol{B}\left(\boldsymbol{r}_{Q_{i}}\right)-\boldsymbol{B}\left(\boldsymbol{r}_{P_{j}}\right)=-\mu_{r j} \boldsymbol{B}_{0}\left(\boldsymbol{r}_{P_{j}}\right)
$$

After solving the matrix system above, the magnetic flux density of each tetrahedral element can be obtained. The induced magnetic flux density $\boldsymbol{B}$ at an arbitrary point $P$ around the ferromagnetic object can be calculated based on Eq. (5).

\section{Magnetic field calculation based on Hybrid Mesh IEM}

Discreting the ferromagnetic object is the basis of Eq.(4) to obtain $\boldsymbol{B}_{\boldsymbol{i}}$ at barycenter of each element and different mesh elements can be chosen, such as tetrahedral element and hexahedral element. However, the two kinds of mesh elements mentioned above have their own advantages [19]. For different parts of 3D objects, the mesh quality can be improved effectively by considering the mixed mesh strategy, especially for complex shaped 3D objects. Two key problems for 3D magnetostatic field computation with surface integral equation method coupling coefficient matrixes construction and $\boldsymbol{B}_{\boldsymbol{i}}$ solving of each element. As far as ferromagnetic objects are discreted with mixed elements, coupling coefficient matriexs should be calculated with tetrahedral elements and hexahedral elements, respectively.

\section{Coupling coefficient matrixs of hexahedral elements}

Information (number of nodes, number of elements and coordinates of nodes) of each mesh element can be obtained after hybrid mesh. In general, an ordinary irregular hexahedral element shown in Fig. 2 can be taken as the study object to demonstrate how to get the coupling coefficient matrix

of

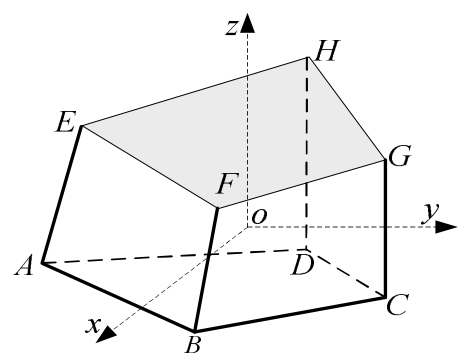

Fig. 2 The irregular hexahedral element

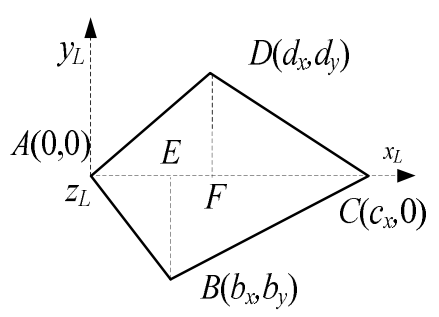

Fig.3 The local reference frame of the surface $s_{l}$ of the irregular hexahedral elemen

With Eq.(3), it is easy to know that three kinds of surface integrals (Eqs.(5) (7)) are the key problems to get coupling coefficient matrix of each element according to information from mesh elements.

$$
\begin{aligned}
& s_{l x}=\int_{s_{l}} \frac{x_{P}-x_{Q_{l}}}{\left|\boldsymbol{r}_{P Q_{l}}\right|^{3}} d s_{Q} \\
& s_{l y}=\int_{s_{l}} \frac{y_{P}-y_{Q_{l}}}{\left|\boldsymbol{r}_{P Q_{l}}\right|^{3}} d s_{Q} \\
& s_{l z}=\int_{s_{l}} \frac{z_{P}-z_{Q_{l}}}{\left|\boldsymbol{r}_{P Q_{l}}\right|^{3}} d s_{Q}
\end{aligned}
$$

where $S_{l}$ denotes the $l_{t h}$ surface of a hexahedron $(l=1,2, \mathrm{~L}, 6),\left(x_{p}, y_{p}, z_{p}\right)$ is the coordinates of field point $P$ in coordinate system of ferromagnetic object. Shape and position of hexahedral elements are always different because of the different mesh proposals. In the global coordinate system (coordinate system of the vessel), the surface integrals above are difficult to be calculated immediately. Herein, building local coordinate system $x_{L} y_{L} z_{L}$ (Shown in Fig.3) on each surface of the mesh element makes surface integrals easy to be obtained. 
In terms of calculating surface integrals, the numerical integral method [20] and analytical integral method [13] could be selected. As the numerical integral method may result in considerable errors in some integral points, the analytical integral method is chosen here and this method has been introduced detailedly in Ref.[19]. For $s_{l x}$ calculating, integral contributions of triangle $\boldsymbol{A B C}$ and $\boldsymbol{A C D}$ should be calculated and labeled with $\triangle A B C_{x}$ and $\triangle A C D_{x}$, respectively. To calculate $s_{l y}$, the integral contributions of triangle $\boldsymbol{B C E}, \boldsymbol{A E B}, \boldsymbol{C F D}$ and $\boldsymbol{A F D}$ should be computed (labeled with $\triangle B C E_{y}, \triangle A E B_{y}, \triangle C F D_{y}$ and $\triangle A F D_{y}$, respectively). For $s_{l z}$, the integral contributions of triangle $\boldsymbol{A B C}$ and $\boldsymbol{A C D}$ should be calculated and labeled with $\triangle A B C_{z}$ and $\triangle A C D_{z}$, respectively.

\section{Coupling coefficient matrix of tetrahedral elemnents}

The coupling coefficient matrix of an arbitary tetrahedron (shown in Fig.4) can be obtained by using a similar method with irregular hexahedral element. To calculate $S_{l x}$, integral contributions of triangle $\boldsymbol{A B C}$ should be calculated and labeled with $\triangle A B C_{x}$. For $s_{l y}$, the integral contributions of triangle $\boldsymbol{A} \boldsymbol{E B}$ and $\boldsymbol{C E B}$ should be solved and labeled with $\triangle A E B_{y}$ and $\triangle C E B_{y}$, respectively. Then the integral contribution of triangle $\boldsymbol{A B C}$ should be obtained (labeled $\triangle A B C_{z}$ ) to calculate $S_{l z}$. Therefore, $S_{l x}, S_{l y}$ and $S_{l z}$ can be expressed as,

$$
\begin{aligned}
& S_{l x}=\Delta A B C_{x} \\
& S_{l y}=\Delta A E B_{y}+\Delta C E B_{y} \\
& S_{l z}=\Delta A B C_{z}
\end{aligned}
$$

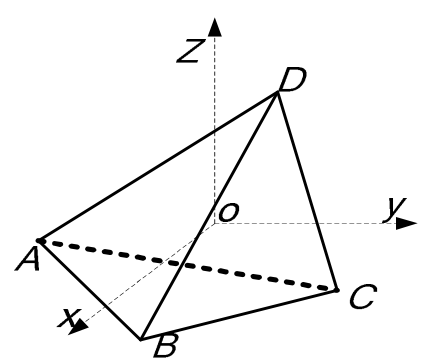

Fig.4 Common tetrahedral element

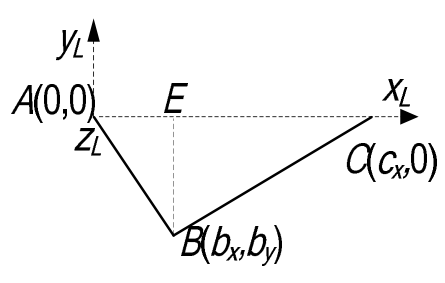

Fig.5The local coordinate system of the $\boldsymbol{s} l$ of tetrahedral surface

\section{Calculations and Verifications}

In this article, two examples are considered to verify the proposed algorithm. One is the magnetostaic modeling of an irregular object magnetized by uniform external magnetic field. The other is a simple experiment that is executed with a steel plate placed in geomagnetic field environment.

\section{Simulation example}

As shown in Fig. 6, a solid, irregularly shaped iron object with a relative permeability of 150 is placed into an external uniform field $B_{0}=34500 e_{z} n T$. The coordinates of the points in Fig.6 are $\boldsymbol{A}(-10,-50,-10), \boldsymbol{B}(-10,-50,10), \boldsymbol{C}(10,-50,-10), \boldsymbol{D}(10,-50,10), \boldsymbol{E}(10,20,10), \boldsymbol{F}(0,80,0)$, $\boldsymbol{P}_{1}(0,-100,20), \boldsymbol{P}_{2}(0,100,20) .201$ field points are arranged along Linel (Shown in Fig.6, from $\mathrm{P}_{1}$ to $\mathrm{P}_{2}$ ) and Line2 (from $\mathrm{P}_{3}$ to $\mathrm{P}_{4}$ ) equally. Meshed with hybrid elements (1672 tetrahedrons and 1225 hexahedrons) and 2104 mesh nodes by software HYPERMESH, three-component magnetic flux intensity of the irregular object are calculated by the proposed hybrid mesh IEM.

In order to verify accuracy of the proposed hybrid mesh IEM, magnetic flux intensity are also calculated by FEM software Flux3D (labeled Flux $\times$ ), which are used to compare with that of the proposed hybrid mesh IEM (labeled Mix $\times$ ). In Figs. 7 Fig.12, X-component, Y-component and 
Z-component of magnetic flux intensity of Line1 and Line 2 are demonstrated in detail. Herein, the actual $\boldsymbol{B}_{\boldsymbol{x}}$ of Line1 and Line2 are zero, and in Fig.7 and Fig.8, $B_{x}$ of the filed points are are close to zero. In fact, those errors of $B_{x}$ can be smaller if the number of mesh elements increases obviously. If $B_{y}$ and $B_{z}$ of the simulation with Flux3D are considered as references, the differences of $B_{y}$ and $B_{z}$ of the proposed hybrid mesh IEM could be calculated and the maximum of them are less than $2.10 \%$.

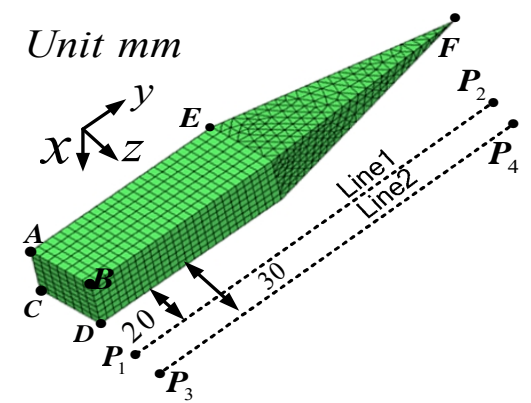

Fig.6 The irregular solid ferromagnetic object

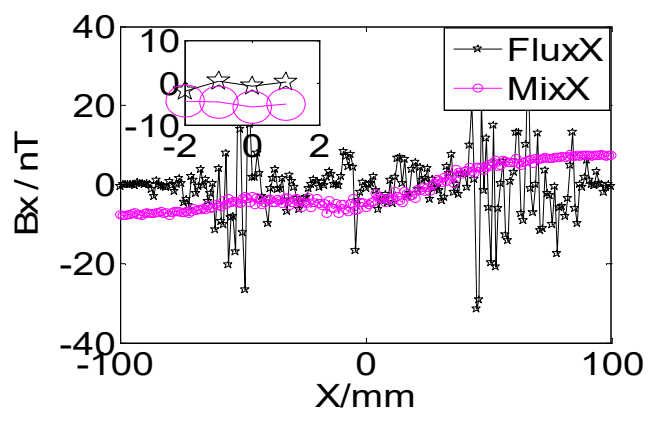

Fig.7 $B x$ of Line1

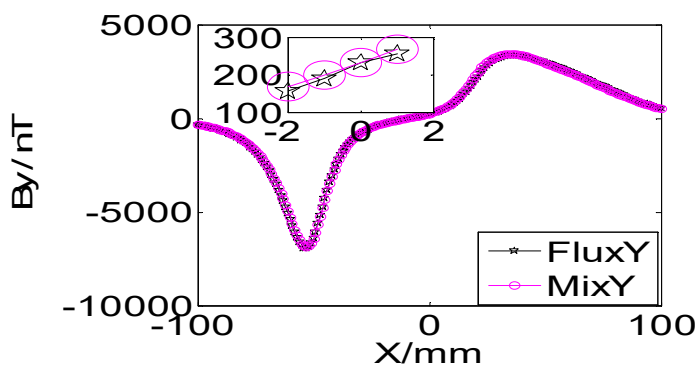

Fig.9 By of Line1

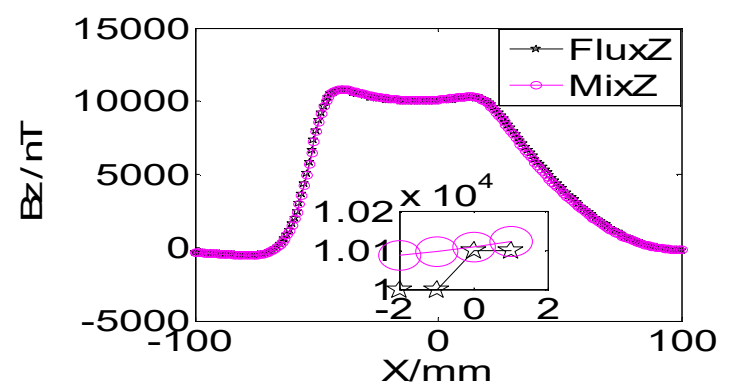

Fig.11 $\mathrm{Bz}$ of Line 1

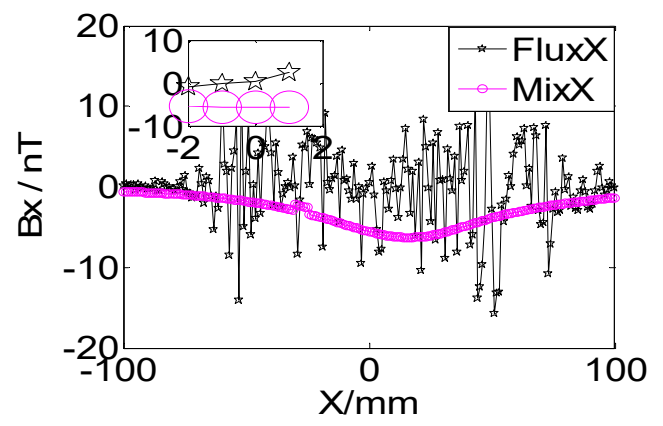

Fig. 8 Bx of Line2

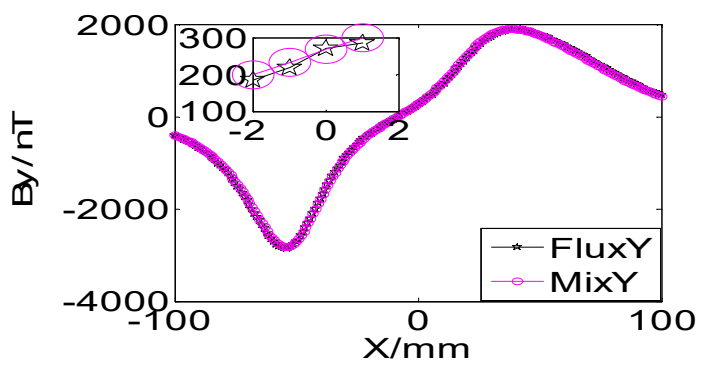

Fig.10 By of Line2

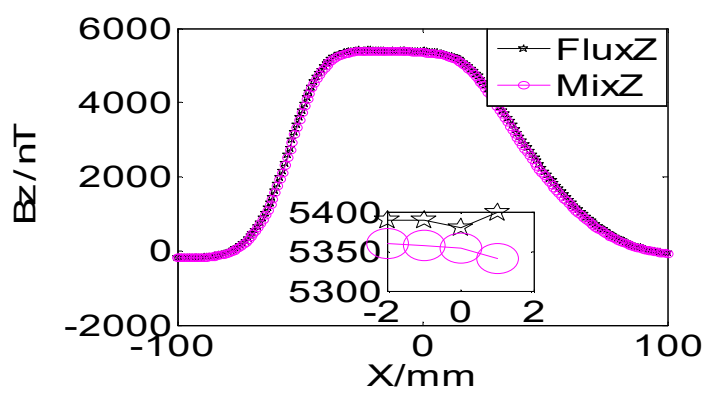

Fig.12 $B z$ of Line2

\section{Experimental example}

As shown in Fig. 14, a steel plate with relative permeability 100 , length $1000 \mathrm{~mm}$, width $200 \mathrm{~mm}$ and thickness $27 \mathrm{~mm}$ is immerged into geomagnetic field with horizontal component $34484.6 \mathrm{nT}$ and vertical component 34800nT. The 31 field points are located in Line3 (from $\mathrm{P}_{1}$ to $\mathrm{P}_{2}$ ) uniformly. In the laboratory, the steel plate is placed on the nonmagnetic carrier on a track. Three triaxial magnetic sensors are placed under the track in a line vertical to the track (one sensor in the middle of the line is displayed in Fig.14). The axes of the triaxial magnetic sensors are arranged as the same as the steel 
plate coordinates system which is depicted in Fig.13. When the nonmagnetic carrier moves over the magnetic sensors, the magnetic anomaly of the steel can be recorded. To eliminate the influence of the permanent magnetic field, the steel plate is placed in four different courses when measuring. Based on the measured field in four courses, the measured induced field with longitudinal and vertical magnetization produced by horizontal component of the geomagnetic field can be obtained, which are used to compare with the calculated field.

In terms of numerical calculations, the steel plate is discreted with 2462 mesh nodes and hybrid elements (shown in Fig.13, 3737 tetrahedrons and 812 hexahedrons). Meanwhile, three-component magnetic flux intensity are calculated by Flux3D. In Fig.14 and Fig.15, normalized measured values (labeled Mea $\times$ ), normalized values of hybrid mesh IEM (labeled NORMAL $\times$ ) and normalized values of simulations with Flux (labeled Flux $\times$ ) of $B_{x}$ and $B_{z}$ are displayed. Thus, maximum differences of numerical results obtained by Flux3D and the proposed hybrid mesh IEM are less than $3.20 \%$ when the values of experiments are taken as references.

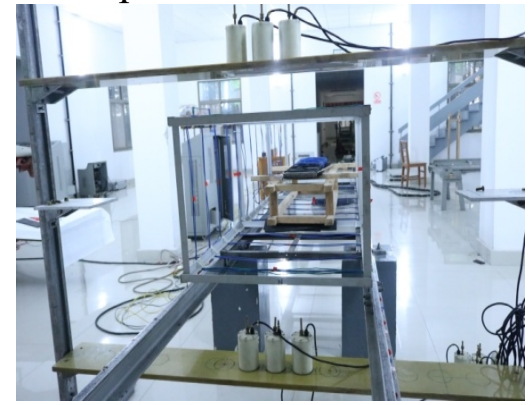

Fig.13 The magnetic field measurement of the thin steel plate

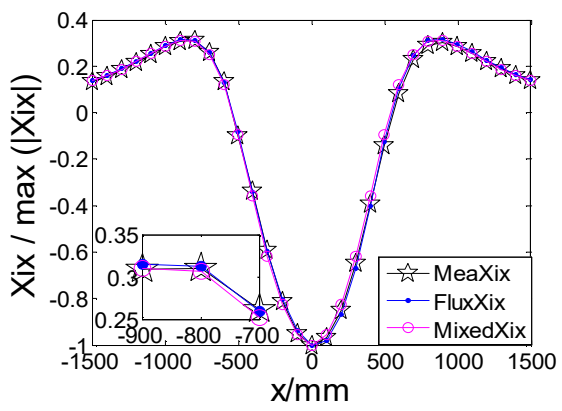

Fig.15 normalized Xix of line3

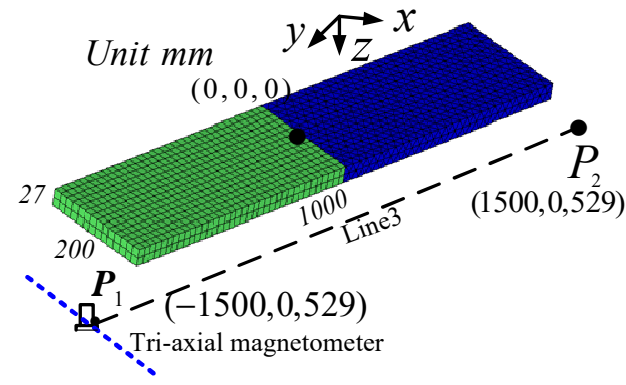

Fig.14 The sketch map of the magnetic field measurement of thin steel plate

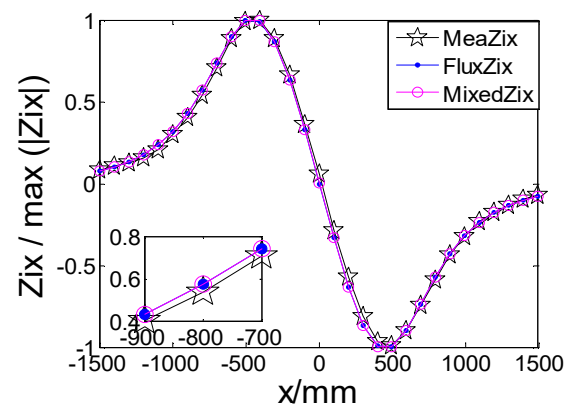

Fig.16 normalized Zix of line3

\section{Conclusion}

Mesh quality of ferromagnetic objects is a key factor which largely influences the computational precision and efficiency of the magnetism integral equation method. For the complex shaped 3D ferromagnetic objects, combing advantages of different kinds of mesh elements, such as tetrahedral element and hexahedral element, is an effective way to improve the mesh quality. Simulation and experimental examples demonstrate the reliable accuracy of the proposed hybrid mesh IEM. In future work, the good mesh strategy and the fast computational methods such as FMM will be studied to integrate with the proposed hybrid mesh method in this article, which would be applied to solve large-scale magnetostatic problems efficiently.

\section{Acknowledgements}

This work is supported in part by National Natural Science Foundation of China under grant No. 41476087 and No. 51377165. 


\section{References}

[1] Holmes. J. J., Exploitation of a Ship's Magnetic Field Signatures (Synthesis Lectures on Computational Electromagnetics), Morgan and Claypool Publishers, San Rafael, California, 2006.

[2] Holmes. J. J., Reduction of a Ship's Magnetic Field Signatures (Synthesis Lectures on Computational Electromagnetics), Morgan and Claypool Publishers, San Rafael, California, 2008.

[3] Pang, H. F., Zhang, Q., Li, J., et al., "Improvement of vector compensation method for vehicle magneticdistortion field, ”J. Magn. Magn. Mater.,Vol. 353 , No., , 130-134, 2014.

[4] Kildishev, A. V., Nyenhuis, J. A., "External magnetic characterization of marine vehicles, ”OCEANS 2000 MTS, Vol.2 , 1145-1147, 2000.

[5] Gao, J. J., Zhu, X. L., Zhu, W. B., "Accurate calculating method of marine three-component geomagnetic field in shipboard measurement, "Chinese Automation Congress, 1504-1507, 2015.

[6] Woloszyn, M., “Analysis of aircraft magnetic interference, "International Joural of Applied Electromagnetics and Mechanics, Vol. 39, No.1-4, 129-136, 2012.

[7] Woloszyn, M., “Analysis of aircraft magnetic interference, "International Joural of Applied Electromagnetics and Mechanics, Vol. 39, No.1-4, 129-136, 2012.

[8] Brunotte, X., Meunier, G., and Bongiraud, J., "Ship magnetization modeling by the finite element method, ’IEEE Trans. Mag., Vol.29, No.2, 1970-1976, 1993.

[9] Damidau, F., Bandelier, B., and Penven, P., "A fast and precise determination of the static magnetic field in the presence of thin iron shells," IEEE Trans. Mag., Vol.31, No.6, 3491-3493, 1995.

[10] Chadebec, O., Coulomb, J. L., Bongiraud, J., et al, "Recent improvements for solving inverse magnetostatic problem applied to thin shells, ”IEEE Trans. Mag., Vol.38, No.2, 1005-1008, 2002.

[11] Chadebec, O., Coulomb, J. L., Leconte, V., et al., "Modeling of static magnetic anomaly created by iron plates, "IEEE Trans. Mag., Vol.36, No.4, 667-671, 2000.

[12] Chadebec, O., Coulomb, J. L., Cauffet, G., et al., "How to well pose a magnetization identification problem, ’IEEE Trans. Mag., Vol.39, No.43, 1634-1637, 2003.

[13] Chadebec, O., Coulomb, J. L., and Janet, F., “A review of magnetostatic moment method, "IEEE Trans. Mag., Vol.42, No.4, 515-520, 2006.

[14] Rodger, D., Leonard, P. J., and Lai, H. C., "Surface elements for modelling 3D fields around thin iron sheets , "IEEE Trans. Mag., Vol.29, No.2, 1483-1486, 1993.

[15] Zhao, K. Z., Vouvakis, M., Lee, J. F., "The adaptive cross approximation algorithm for accelerated method of moment computations of EMC problems, "IEEE Trans. Mag., Vol.47, No.4, 763-773, 2005.

[16] Zhou, G. H., Liu, S. D., Xiao, C. H., and Liu, D. M., "Approach for a fast computation of induced magnetic field created by naval vessels, "International Journal of Applied Electromagnetics and Mechanics, Vol.47, No.3, 753-764, 2015.

[17] Zhang, M., Guo, L., “A fast algorithm for pre-procedure of method of moments, ”Journal of Nanjing University of Posts and Telecommunications:Natural Science Edition, Vol.30, No.5, 95-97, 2010. (in Chinese)

[18] Fan, M. W., Yan W. L., Integral equation method in electromagnetics, China Machine Press, Beijin, 1988.

[19] Zhou, G. H., Xiao, C. H., Liu, S. D., et al., “3D magnetostatic field computation with hexahedral surface integral equation method," Tansactions of China Electrotechnical Society, Vol.24, No.3, 1-7, 2009. (in Chinese text) 
[20] Kwon, O. M., Surussavadee, C., Chari, M. V. K., at al, "Analysis of the far field of permanent-magnet motors and effects of geometric asymmetries and unbalance in magnet design," IEEE Trans. Mag., Vol.40, No.2, 435-442, Mar.2004.

[21] Günter, L., Electromagnetic Field Theory for Engineers and Physicists, Springer, New York, 2010 . 